\title{
Quantum Groups and WZNW Models
}

\author{
A. Alekseev and S. Shatashvili^ \\ Leningrad Steklov Mathematical Institute, Fontanka 27, SU-191011, Leningrad, USSR
}

Received December 27, 1989

\begin{abstract}
An explanation of the appearance of quantum groups in chiral WZNW models is given. Invariance of the theory under quantum group action is discussed.
\end{abstract}

\section{Introduction}

Recent developments of various approaches to conformal field theory have led to a deep understanding of the properties of rational conformal field theories (RCFTs). It was shown [1] that monodromy properties of conformal blocks in the WZNW model defines braiding matrices which are closely related to the theory of quantum groups [2]. A category-theoretic point of view on conformal field theory developed by Moore and Seiberg [3] gives evidence that these two subjects, RCFT and the theory of quantum groups are very similar. On the other hand, E. Witten [4] introduced a universal language both for the conformal field theory and integrable lattice models, where the quantum group plays a key role. But until now, the above observation i.e., coincidence of monodromy properties of conformal blocks in $S U(n)$ WZW model with braiding matrices of $U_{q}(s l(n))$ has been rather mysterious and in need of a conceptual explanation.

In this paper we'll give an explanation of the appearance of $R$-matrices in conformal field theory and discuss the meaning of invariance of the theory under the quantum group. Our idea is based on the geometric approach to the conformal field theory [5-7]. The main point of our interpretation is that in the chiral WZNW model the dynamics of the element of the loop group, $g(x)$, completely defines the theory and therefore the quantum group must appear at the classical level as a corresponding Poisson-Lie group, which acts on the Poisson bracket relations of the loop group element. $g(x)$ itself after quantization contains vertex operators of the theory (see, i.e., $[6,8]$ ) and thus quantization of the above Poisson brackets [or, the same, quantum exchange algebra for $g(x)]$ must describe the monodromy

* Visitor at the Enrico Fermi Institute, 5640 S. Ellis Ave. Chicago, IL 60637. Supported by NSF-PHY-86-57788 
properties of conformal blocks. It means that first we need to define the Poisson bracket on the loop space, i.e., $\left\{g(x)^{\otimes}, g(y)\right\}$.

It was shown in [7] that the geometric quantization of the "model space" for the Kac-Moody and the Virasoro groups reproduces the chiral part of RCFT. The construction of the model space is based on the Kirillov-Kostant 2-form $\Omega$ on the coadjoint orbit of the Lie group $G$. If the point of the coadjoint orbit is parametrized by the group element, then the 2 -form $\Omega$ defines a degenerate symplectic structure on the group itself, thus it gives multivalued Poisson brackets on group elements. But field theory (i.e., the case of the infinite dimensional Lie group) is defined not only by the Lagrangian which is equal to $d^{-1} \Omega$, but also by the boundary conditions on the input fields. Therefore we must specify the boundary conditions on the field $g(x)$, i.e., we can fix the values of several matrix elements at $x=0$ and $x=2 \pi$. In Sect. 2 of this paper we explain that the "right" choice of boundary conditions guarantees the univaluedness of the Poisson brackets for the elements of the loop group, but at the same time it breaks the "isotopic symmetry" of the theory. The Jacobi identity on $\left\{g(x),{ }^{\otimes} g(y)\right\}$ and the choice of natural boundary conditions will lead us to $r$-matrix Poisson brackets. We'll discuss two types of boundary condition, which, as will be demonstrated in Sect. 3, are related to IRF and vertex pictures of the quantum theory. In one case we'll get an $r$-matrix which satisfies the classical Yang-Baxter equation, in another, the corresponding $r$-matrix depends on quasi-momentum and it is very similar to the well-known $r$-matrix of [9]; it satisfies the classical YB equation in the IRF picture. These two $r$-matrices are closely related to each other in our approach, because the corresponding boundary conditions are related to a linear differential equation and thus there must be a linear relation between $g(x)$ in these two pictures (see below Sect. $1 \mathrm{~b}$; this linear transformation is similar to one introduced by Babelon $[10]$ in Liouville theory $[9,11]$. This is not mysterious, because, as was shown in $[6,12]$, Liouville theory is a Drinfeld-Sokolov Hamiltonian reduction from $S L(2, R)$ Kac-Moody theory and in particular the Poisson brackets of input field $F(x): F(x) \in \operatorname{Diff} S^{1}$, defined by the gravitational WZ action is of $r$-matrix nature with $S L(2) r$-matrix) [7]. At the quantum level this linear transformation corresponds to the relation between IRF and vertex pictures [13,14]. At the end of Sect. 2, we discuss the action of the Poisson-Lie group and one possible interpretation of the invariance of the theory under Poisson-Lie group.

In Sect. 3 we quantize the classical exchange algebra using the free field parametrization of the chiral WZNW model of $[7,8]$. Instead of the $r$-matrix Poisson brackets we will have a quantum exchange algebra which is of $R$-matrix form. The quantum $R$-matrix corresponds to that of $S L_{q}(2)$ with deformation parameter $q=\exp \left[\frac{2 \pi i}{K+2}\right]$. Each column of the quantum matrix $g(x)$ is in a representation of the Kac-Moody group realized in bosonic modules with spin $\frac{1}{2}$ but with different "charges" in Feigin-Fuchs-Dotsenko-Fateev language. We explain that the quantum group acts in the row of the matrix $g(x)$, i.e., between the columns - in the space of several copies of the representation of the Kac-Moody group, with different charges. In this sense these two objects, the Kac-Moody group and the quantum group, are simultaneously represented in the loop group element $g(x)$. [The generalization to the case of any spin is straightforward; we can write the matrix elements of $g(x)$ in the spin $j$ representation as symmetric 
polynomials of the matrix elements of spin $\frac{1}{2} g(x)$.] After the quantization, in the case of the compact group (the non-compact case is similar to the Virasoro one in [7]), the theory contains a finite number of Kac-Moody representations (so-called "integrable representations"), which correspond in our approach to the quantization of quasi-momentum; at the same time there is a quantum group spin, therefore in the corresponding RCFT only a finite number of quantum group representations are present for $q$ a root of unity. At the end of Sect. 3 the relation between the generators of the quantum exchange algebra and the Kac-Moody generators is discussed.

In Sect. 4 the quantum group is derived as a right-hand symmetry of the WZNW model. It is shown that the currents defined in Sect. 3 are invariant under the action of the quantum group.

\section{Poisson-Lie Group in Classical WZW Model}

Poisson-Lie group is a classical analog of a quantum group. Therefore it is quite natural to expect its appearance in the classical chiral WZW theory, where instead of the quantum exchange algebra we have to deal with the Poisson brackets and symplectic structures. In the present approach it is easy to consider the general case of $S L(n)$ without any technical problems.

\section{a) Poisson Brackets in SL(n) WZW Model}

It is known that the Kirillov-Kostant 2-form $\Omega$ on the coadjoint orbit of the KacMoody group is closely related to the WZW model. More precisely, $d^{-1} \Omega$ written in group variables exactly coincides with the WZW Lagrangian:

$$
d \alpha=\Omega=\frac{K}{4 \pi} \int_{0}^{2 \pi} d x \operatorname{Tr}\left\{g^{-1} d g \partial\left(g^{-1} d g\right)\right\}
$$

where $K$ is the central charge of the Kac-Moody algebra $\partial \equiv \frac{\partial}{\partial x}, x \in S^{1}, g \in L G$. The restriction of $\Omega$ on the matrices $g(x)$ with fixed monodromy properties $g^{-1}(0) g(2 \pi)$ $=M=$ const defines the nondegenerate symplectic structure on the corresponding coadjoint orbit and thus defines the Poisson brackets of the linear functions on the orbit; i.e., for currents

$$
\left\{J^{a}(x), J^{b}(y)\right\}=2 \pi\left(f^{a b c} J^{c}(x) \delta(x-y)-K \delta^{a b} \delta^{\prime}(x-y)\right) .
$$

Here $J^{a}(x)=\operatorname{Tr}\left\{J T^{a}\right\}, J=K \partial g \cdot g^{-1}$, and $T^{\alpha}$ are generators of the Lie group $G$ [in this section $G=S L(n)]$. On the loop group itself the 2 -form $\Omega$ is degenerate, i.e., in the case of boundary conditions $g^{-1}(0) g(2 \pi)=M$ it doesn't contain the differentials along $H$, where $H$ is a Cartan subgroup, defined by $M$.

In this section we give two types of the construction of the Poisson brackets on the loop group, or the same, construction of nondegenerate symplectic structures on $L G$, which are naturally related to the WZNW model. These two versions correspond to different choices of boundary conditions on the element of loop group $g(x)$. Before describing the boundary conditions let us give a formal inverse of the symplectic form $\Omega$, which is given by the multivalued Poisson bracket

$$
\left\{g(x)^{\otimes}, g(y)\right\}=-\frac{\pi}{K} g(x) \otimes g(y)(C \in(x-y)+X),
$$


where $C=T^{a} \otimes T^{a}$ and $X$ is some constant matrix, the anulator of the symplectic form; it must be defined from the boundary conditions. On the other hand, it means that the value of $X$ is related to the additional term in $\Omega$, which is necessary to introduce when we have imposed the boundary condition on $g$.

The formal relation (3) is easy to get from the 2 -form $\Omega$. Here we must use the scheme

$$
\Omega=\frac{1}{2} d \xi^{a} \Omega_{a b} d \xi^{b} \Rightarrow\left\{\xi^{a}, \xi^{b}\right\}=\left(\Omega^{-1}\right)^{b a} .
$$

But in our case the coordinates $\xi=g(x)$ aren't independent $(\operatorname{det} g=1, g \in S L(n))$ and therefore it is convenient to consider $\tilde{g}(x) \in G L_{+}(n)$, $\operatorname{det} \tilde{g}>0, \tilde{g}=\lambda g$, where $\lambda \in R_{+}$. Then

$$
\Omega(\tilde{\mathrm{g}})=\Omega(\mathrm{g})+\frac{K n}{4 \pi} \int_{0}^{2 \pi} d(\log \lambda) \partial[d(\log \lambda)] d x .
$$

Now in the 2-form $\Omega(\tilde{g})$ the coordinates $\tilde{g}_{i j}$ are independent and we can use (4):

$$
\left\{\tilde{g}_{i j}(x), \tilde{g}_{k l}(y)\right\}=-\frac{\pi}{K}\left[\tilde{g}_{i l}(x) \tilde{g}_{k j}(y) \varepsilon(x-y)+\tilde{g}_{i m}(x) \tilde{g}_{k n}(g) X_{j l}^{m n}\right],
$$

with $X_{j l}^{m n}=-X_{l j}^{n m}$ is constant matrix, the anulator of $\Omega$. It follows from the factorization property of $\Omega$ [Eq. (5)], that

$$
\left\{\tilde{g}_{i j}(x), \tilde{g}_{k l}(y)\right\}=\left\{g_{i j}(x), g_{k l}(y)\right\} \lambda(x) \lambda(y)+g_{i j}(x) g_{k l}(y)\{\lambda(x), \lambda(y)\} .
$$

Poisson bracket for $\lambda$ is defined from Eq. (5),

$$
\{\lambda(x), \lambda(y)\}=-\frac{\pi}{K n} \lambda(x) \lambda(y) \varepsilon(x-y) .
$$

Now from Eqs. (6), (7), and (8), it follows that $\left\{g_{i j}(x), g_{k l}(y)\right\}$ acquires the form:

$$
\left\{g_{i j}(x), g_{k l}(y)\right\}=-\frac{\pi}{K}\left[\left(g_{i l}(x) g_{k j}(y)-\frac{1}{n} g_{i j}(x) g_{k l}(y)\right) \varepsilon(x-y)+g_{i m}(x) g_{k n}(y) X_{j l}^{m n}\right],
$$

or in tensor notation

$$
\left\{g(x)^{\otimes} g(y)\right\}=-\frac{\pi}{K} g(x) \otimes g(y)\left[\left(P-\frac{1}{n} I \otimes I\right) \varepsilon(x-y)+X\right],
$$

where $P$ is a permutation matrix $P_{i j}^{k l}=\delta_{i}^{l} \delta_{j}^{k}$ and the traceless combination $P-\frac{1}{n} I \otimes I$ coincides with Casimir operator $C=T^{a} \otimes T^{a}=P-\frac{1}{n} I \otimes I$. Thus Poisson brackets of $g(x)$ are given by the multivalued expression Eq. (3). Let us mention that the main part of Eq. (3) is right invariant, i.e., it is invariant under the transformation $g(x) \rightarrow g(x) \cdot h, h \in G$, but the presence of the anulator $X$ can break this symmetry.

Now let us calculate the matrix $X$ in the case of the "initial-final" boundary condition:

$$
\begin{array}{cc}
g_{m n}(0)=0 ; & n>m, \\
g_{m n}(2 \pi)=0 ; & n<m .
\end{array}
$$


There are two conditions that we must check: 1 . the constraints $(10)$ must be first-class; 2. the Jacobi identity. It is easy to show that condition 1 is equivalent to:

$$
\begin{array}{cc}
C_{j l}^{m n}+X_{j l}^{m n}=0, & n<l, \\
-C_{j e}^{m n}+X_{j l}^{m n}=0, & n>l .
\end{array}
$$

This equation defines $X$ up to diagonal elements, but these terms are irrelevant, because they can be absorbed by the corresponding redefinition of $g$. Thus we have from Eq. (11)

$$
X=\sum_{\alpha>0}\left(T_{-\alpha} \otimes T_{\alpha}-T_{\alpha} \otimes T_{-\alpha}\right)
$$

where summation in Eq. (12) is over positive roots of $S L(n)$ and $T_{\alpha}=E_{i j}(i<j)$. Finally, the Poisson bracket for the case of boundary conditions in Eq. (10), acquires the form:

$$
\left\{g(x)^{\otimes}, g(y)\right\}=-\frac{\pi}{K} g(x) \otimes g(y)\left[r^{+} \theta(x-y)+r^{-} \theta(y-x)\right],
$$

where

$$
\begin{gathered}
r^{+}=C+X=\sum_{i} H_{i} \otimes H_{i}+2 \sum_{\alpha>0} T_{-\alpha} \otimes T_{\alpha}, \\
r^{-}=-C+X=-\sum_{i} H_{i} \otimes H_{i}-2 \sum_{\alpha>0} T_{\alpha} \otimes T_{-\alpha},
\end{gathered}
$$

$H_{i}$ is a basis in Cartan subalgebra. $r^{+}$and $r^{-}$are the solution of the classical YangBaxter equation $r^{+}-r^{-}=2 C ; X=\frac{r^{+}+r^{-}}{2}$.

Let us mention that Poisson bracket (13) is left-invariant, but not rightinvariant. The reason is that 2 -form $\Omega$ is degenerate on the loop space; boundary condition (10) makes it nondegenerate but noninvariant.

\section{b) Free Field Variables in WZW Model}

Recently a useful method for the treatment of Kac-Moody algebra, so-called bosonization, was introduced [15, 6, 8, 16-18]. In this parametrization the Lagrangian of the WZW model is quadratic and the currents of the Kac-Moody algebra are polynomials of free fields; these free fields are "Darboux" variables for the symplectic form $\Omega[6,8]$. For simplicity, we consider the case of $S L(2)$; the general case of $S L(n)$ can be investigated using [8]. The classical exchange algebra (13) for the case of $S L(2)$ using the free field parametrization of $g[6,8]$ first was obtained by Block [19] (see also the appendix in [7]).

The classical action in the WZW model is defined by the 2-form $\Omega$ as $W(g)=\int \alpha$ $=\int d^{-1} \Omega$. It was shown in $[6,8]$ that the action can be written in "Darboux" variables as

$$
W(g)=-\frac{K}{2 \pi} \int[\partial \phi d \phi+\omega d \chi] d x
$$


where the element of the loop group $g(x)$ in terms of free bosonic fields $\phi, \omega, \chi$ acquires the form:

$$
g=\left(\begin{array}{ll}
1 & 0 \\
\chi & 1
\end{array}\right)\left(\begin{array}{cc}
e^{-\phi} & 0 \\
0 & e^{\phi}
\end{array}\right)\left(\begin{array}{ll}
1 & \psi \\
0 & 1
\end{array}\right)
$$

with the condition $\partial \psi=\omega e^{2 \phi}$. Quantum action differs from the classical one (15) by the renormalization of the kinetic term for $\phi$ :

$$
W_{q}(g)=-\frac{1}{2 \pi} \int[(K+2) \partial \phi d \phi+K \omega d \chi] d x
$$

which is a consequence of the anomaly of the measure in the path integral (see [8]). (We discuss the quantum case in Sect. 3.) We can introduce boundary condition different from (10) by fixing the monodromy matrix $\mathscr{M}(t)=g^{-1}(0, t) g(2 \pi, t)$. The case of constant $\mathscr{M}$ corresponds to the coadjoint orbit whereas the case where $\mathscr{M}(t)$ is considered as a dynamical variable corresponds to the so-called "model space" [7]. Let us assume that the monodromy matrix $\mathscr{M}$ is diagonal:

$$
\mathscr{M}=\left(\begin{array}{cc}
M^{-1} & 0 \\
0 & M
\end{array}\right) .
$$

This means then that we have the following properties for the free fields

$$
\begin{gathered}
\chi(2 \pi)=\chi(0), \\
e^{\phi(2 \pi)}=M e^{\phi(0)} ; \quad \phi(2 \pi)=\phi(0)+2 \pi m, \\
\psi(2 \pi)=M^{2} \psi(0) ; \quad \omega(2 \pi)=\omega(0),
\end{gathered}
$$

where $m=\frac{1}{2 \pi} \log M$. One can obtain that the zero mode $\phi_{0}$ of

$$
\phi=\phi_{0}+m x+\sum \phi_{n} e^{i n x}
$$

is in the kernel of the symplectic form because it is presented in the action only as a total derivative

$$
\Delta S\left(\phi_{0}\right)=-K \int m d \phi_{0} .
$$

It means that the corresponding orbit is $\widehat{L G} / H$, where $G=S L(2)$ and $H$ is maximal torus - i.e. the Cartan subgroup of $S L(2)$ acting from the right-hand side.

It is easy to extend the symplectic 2 -form $\Omega$ on the space of matrices with any diagonal monodromy by the addition of the term

$$
\Delta \Omega\left(m, \phi_{0}\right)=-2 K d m d \phi_{0}
$$

which corresponds to the natural Poisson bracket

$$
\{\phi(x), \phi(n)\}=-\frac{\pi}{2 K} \varepsilon(x-y) .
$$

Now we have the Poisson brackets for the variables $g_{i j}(x)$ with diagonal monodromy; these "Darboux" variables are

$$
\begin{gathered}
\omega(x)=\sum_{n} \omega_{n} e^{i n x}, \quad \chi(x)=\sum_{n} \chi_{n} e^{i n x}, \\
\phi(x)=\phi_{0}+m x+\sum_{n} \phi_{n} e^{i n x}
\end{gathered}
$$


with non-trivial Poisson brackets

$$
\begin{gathered}
\left\{\omega_{n}, \chi_{-n}\right\}=-\frac{1}{K}, \\
\left\{\phi_{n}, \phi_{-n}\right\}=-\frac{1}{2 \mathrm{inK}}, \quad\left\{m, \phi_{0}\right\}=-\frac{1}{2 K} .
\end{gathered}
$$

Let us give an expression for the field $\psi(x)$ in terms of canonical variables;

$$
\psi(x)=\frac{1}{M^{2}-1}\left[M^{2} \int_{0}^{x} \omega(z) e^{2 \phi(z)} d z+\int_{x}^{2 \pi} \omega(z) e^{2 \phi(z)} d z\right]
$$

or

$$
\psi(x)=\frac{1}{M^{2}-1} \int_{x}^{x+2 \pi} \omega(z) e^{2 \phi(z)} d z
$$

where we mean that $x \in R^{1}$ and the fields $\omega, \chi, \phi$ are given by (22). The integral in $\left(24^{\prime}\right)$ corresponds to the screening operator in the Feigin-Fuchs-Dotsenko-Fateev approach. In our consideration they appeared naturally as components of the field $g(x)$ in the Gauss expansion (16).

Now, using the simple relations (23), (24) it is easy to show that

$$
\{g(x) \otimes(y)\}=-\frac{\pi}{K} g(x) \otimes g(y)\left(r^{+}(M) \theta(x-y)+r^{-}(M) \theta(y-x)\right),
$$

where

$$
\begin{aligned}
r^{+}(M) & =\frac{1}{2}\left(\begin{array}{cccc}
1 & 0 & 0 & 0 \\
0 & -1 & \frac{-4}{M^{2}-1} & 0 \\
0 & \frac{4 M^{2}}{M^{2}-1} & -1 & 0 \\
0 & 0 & 0 & 1
\end{array}\right), \\
r^{-}(M) & =\frac{1}{2}\left(\begin{array}{cccc}
-1 & 0 & 0 & 0 \\
0 & 1 & \frac{-4 M^{2}}{M^{2}-1} & 0 \\
0 & \frac{4}{M^{2}-1} & 1 & 0 \\
0 & 0 & 0 & -1
\end{array}\right) .
\end{aligned}
$$

Note that $r^{-}=-\mathrm{Pr}^{+} P$. Compared to (13), $r^{+}\left(r^{-}\right)$now depends on quasi-momentum $M$ which has nontrivial Poisson brackets with the matrix elements of $g(x)$; therefore the Jacobi identity for (25) doesn't coincide with the classical YB equation. This type of $r$-matrix first appeared in the Liouville theory [9]. Let us mention that in the limit $M \rightarrow \infty$ relation (25) acquires the form (13) and now $\psi(x)$ is given by

$$
\psi(x) \stackrel{M^{2} \rightarrow \infty}{\longrightarrow} \int_{0}^{x} \omega(z) e^{2 \phi(z)} d z
$$


which is also the solution of equation $\partial \psi=\omega e^{2 \phi}$ but with the boundary condition $\psi(0)=0$. For this limit the Poisson brackets (25) in the free field parametrizations previously were calculated by Block [19]. The limit $M \rightarrow \infty$ corresponds to the "initial-final" boundary condition (10). In the notation $g=\left(\begin{array}{ll}a & b \\ c & d\end{array}\right)$ the quasiperiodicity condition acquires the form

$$
b(2 \pi)=M b(0), \quad c(2 \pi)=M^{-1} c(0)
$$

and $b(0) \rightarrow 0, c(2 \pi) \rightarrow 0$. As it follows from the Gauss expansion, $g(x)$ is linear in variable $\psi(x)$; thus there must exist a linear transformation which gives the relation between the matrix elements of $g(x)$ in these two pictures, corresponding to the boundary conditions (10) and (18) (compare with [10]).

Let us mention that the Poisson brackets (25) are invariant under the transformation $g(x) \rightarrow g(x) \cdot h$, where $h \in H$ is a constant diagonal matrix. The generator of this transformation is $m=\log M$. Right-hand symmetry under the whole group $G$ is broken; the reason is the choice of diagonal monodromy.

\section{c) Poisson-Lie Group and Symplectic Structure on the Loop Space}

Now we would like to describe a nice trick which allows us to preserve the righthand symmetry. We consider the transformation $g(x) \rightarrow g(x) \cdot h$, where $h \in H$ but with the assumption that on the copy of the group $S L(n)$ some Poisson structure is defined. The condition of invariance of the brackets $\left\{g(x),{ }^{\otimes} g(y)\right\}$ uniquely defines the Poisson brackets for $h$ [20],

$$
\left\{g(x) \cdot h^{\otimes}, g(y) \cdot h\right\}=\{g(x), g(y)\} h \otimes h+g(x) \otimes g(y)\{h, h\} .
$$

From Eq. (27) and (13) we get the expression for $\left\{h{ }^{\otimes} h\right\}$,

$$
\left\{h^{\otimes}, h\right\}=\frac{4 \pi}{K}[r, h \otimes h],
$$

where $r=r^{+}$or $r^{-}\left(r^{+}-r^{-}=2 C\right.$ and commutes with $\left.h \otimes h\right)$. The quadratic Poisson algebra is known as a Sklyanin algebra [21]. The group $G$ with the Poisson brackets (28) is called a Poisson-Lie group and it is the classical analog of a quantum group [in our case $S L_{q}(n)$ ].

Formally it is possible to define the symplectic structure on the whole loop group $\widehat{S L}_{K}(2)$ [generalization to $\widehat{S L}_{K}(n)$ seems to be trivial] with any boundary condition. We already have the Poisson brackets (13) with $g(0)$ lower triangular and $g(2 \pi)$ upper triangular. Let us consider the object $g(x) \cdot h \equiv \tilde{g}(x), h \in S L(n)$. Now $\tilde{g}(x)$ is a matrix with any monodromy and takes value in the whole loop group. But for each $\tilde{g}$ from the loop group there are many representations like

$$
\tilde{g}=g(x) \cdot h=g \mathscr{D}_{D^{-1}} h=g^{\prime}(x) h^{\prime}
$$

with $g^{\prime}(x)$ obeying the same boundary conditions. This means that Poisson structure on $h$ must be invariant under $h \rightarrow \mathscr{D} h$, where $\mathscr{D}$ is a diagonal matrix; [this property is present in (28) because of $\left.\mathscr{D} \otimes \mathscr{D} r^{ \pm}=r^{ \pm} \mathscr{D} \otimes \mathscr{D}\right]$. 
For the case $n=2$ the group element $h$ in terms of Darboux variables acquires the form

$$
h=\left(\begin{array}{ll}
x & y \\
z & t
\end{array}\right),
$$

$x=e^{p}, \quad y=e^{q}, \quad z=\varrho e^{q}, \quad$ and $t=e^{-p}\left(1+\varrho e^{q}\right) \quad$ with symplectic structure $\omega=-\frac{2 \mathrm{~K}}{\pi} d p d q$. From $\{\mathrm{p}, \mathrm{q}\}=-\frac{\pi}{2 K}$ it follows that

$$
\begin{gathered}
\{x, y\}=-\frac{\pi}{2 K} x y, \quad\{t, y\}=\frac{\pi}{2 K} t y, \\
\{x, z\}=-\frac{\pi}{2 K} x z, \quad\{t, z\}=\frac{\pi}{2 K} t z \\
\{x, t\}=-\frac{\pi}{K} y z, \quad\{y, z\}=0 .
\end{gathered}
$$

It is easy to check that $\operatorname{det} h=x y-z t$ is an anulator of the brackets (30) and thus we can impose $\operatorname{det} h=1$. Another anulator is the parameter $\varrho$. Transformation $h \rightarrow \mathscr{D} h$ means that

$$
e^{p} \rightarrow e^{p} \mathscr{D}, \quad e^{q} \rightarrow e^{q} \mathscr{D}, \quad \varrho \rightarrow \mathscr{D}^{-2} \varrho .
$$

$\omega$ is invariant under (31) and therefore we can choose $\varrho^{\prime}=\mathscr{D}^{-2} \varrho=1$; thus $\varrho$ can be excluded from all expressions. Finally, we have the symplectic structure on the whole loop space, i.e., on the space of matrices $g(x)$ with any boundary condition:

$$
\widetilde{\Omega}=\Omega+\omega \text {. }
$$

For the theory defined by this structure the symmetry (27) is ordinary symmetry.

\section{Quantum Exchange Relations}

In the quantum case we must consider the commutation relations defined by the quantum action (17). Our consideration is based on the operator language, which can be easily rewritten on the path integral approach.

\section{a) Exchange Algebra for $a$ and $b$}

In general, the matrix elements $a, b, c$, and $d$ of the loop group element $g=\left(\begin{array}{ll}a & b \\ c & d\end{array}\right)$ are ill defined; they contain free operators at coincident points [see (16)]. But the operators $a$ and $b$ are well-defined for the spin $\frac{1}{2}$ representation of the Kac-Moody group [we'll consider the case of $S L(2)$; generalization to $S L(n)$ is straightforward]:

$$
\begin{gathered}
a(x)=e^{-\phi(x)} \\
b(x)=e^{-\phi(x)} \frac{1}{M^{2}-1} \int_{x}^{x+2 \pi} \omega(z) e^{2 \phi(z)} d z .
\end{gathered}
$$


(Let us mention that after the Drinfeld-Sokolov reduction $[6,12]$ these operators are exactly the vertex operators in minimal model with $\alpha_{+}=\frac{2 \sqrt{a}}{\sqrt{K+2}}$; $K=p / q-2$.) Operator $e^{-\phi(x)}$ we understand as normal ordered in the oscillator picture for $\phi_{n}$ and $\phi_{-n}$. We use the simple exchange relation for the exponents of the free fields

$$
\begin{gathered}
e^{\alpha \phi(x)} e^{\beta \phi(y)}=e^{\beta \phi(y)} e^{\alpha \phi(x)}\left[q^{\frac{\alpha \beta}{4}} \theta(x-y)+q^{-\frac{\alpha \beta}{4}} \theta(y-x)\right], \\
M e^{-\phi(x)}=e^{-\phi(x)} M q^{-1 / 2},
\end{gathered}
$$

with $q=\exp \frac{2 \pi i}{K+2}$.

Thus we have for the first row in $g(x)$ :

$$
V(x) V^{T}(y)=V(y) V^{T}(x)\left[R^{+}(M) \theta(x-y)+R^{-}(M) \theta(y-x)\right],
$$

where $V(x)=\left(\begin{array}{l}a(x) \\ b(x)\end{array}\right), V^{T}(x)=(a(x), b(x)), R^{-}=\left(R^{+}\right)^{-1}$, and $^{1}$ $R^{+}(M)=q^{-1 / 4}$

$$
\left(\begin{array}{cccc}
q^{1 / 2} & 0 & 0 & 0 \\
0 & -q^{-1 / 2} M^{-1} \frac{q^{1 / 2}-q^{-1 / 2}}{q^{1 / 2} M-q^{-1 / 2} M^{-1}} & 1 & 0 \\
0 & \frac{\left(M-M^{-1}\right)\left(q M-(q M)^{-1}\right)}{\left(q^{1 / 2} M-q^{-1 / 2} M^{-1}\right)^{2}} & q^{1 / 2} M \frac{q^{1 / 2}-q^{-1 / 2}}{q^{1 / 2} M-q^{-1 / 2} M^{-1}} & 0 \\
0 & 0 & 0 & q^{1 / 2}
\end{array}\right)
$$

Dynamical considerations using the path integral approach of [7] show that $M$ is quantized in the case of $S U(2): M=q^{l}$, where $l$ is a spin of the Kac-Moody algebra. The non-compact case $S L(2)$ we think can be treated similar to the Virasoro group, as in [7]. Thus after the replacement $M \rightarrow q^{l}, R^{+}$in (35) coincides with the $6 j$ symbols (up to normalization) in the category theory of $S L_{q}(2)$ [22]. The general picture looks as follows: spin of the state $|\varrho\rangle$ is defined by

then

$$
M|\varrho\rangle=q^{l}|\varrho\rangle,
$$

$$
\begin{gathered}
M V_{+}|\varrho\rangle=q^{1 / 2} V_{+} M|\varrho\rangle=q^{l+1 / 2} V_{+}|\varrho\rangle, \\
M V_{-}|\varrho\rangle=q^{l-1 / 2}|\varrho\rangle
\end{gathered}
$$

where $V_{+}=b, V_{-}=a$. It seems that $V_{+}$and $V_{-}$are components of " $3 j$-symbols" for the Kac-Moody algebra, where pairs of indices $\left(\begin{array}{c}j^{\prime} \\ m^{\prime}\end{array}\right),\left(\begin{array}{c}j^{\prime \prime} \\ m^{\prime \prime}\end{array}\right)$ are realized in

${ }^{1} R^{+}$in (35) is very similar to one in [9] in Liouville theory, see also [23] 
bosonized modules. We have

$$
V: \mathscr{H}_{l} \rightarrow \mathscr{H}_{l-1 / 2} \oplus \mathscr{H}_{l+1 / 2}
$$

with

$$
\begin{aligned}
& V_{+}: \mathscr{H}_{l} \rightarrow \mathscr{H}_{l+1 / 2}, \\
& V_{-}: \mathscr{H}_{l} \rightarrow \mathscr{H}_{l-1 / 2} .
\end{aligned}
$$

From the observation that $R^{+}$in (35) coincides with the similar object for the $S L_{q}(2)$ it follows that at the same time $V_{+}$is a $3 j$-symbol for the quantum group $S L_{q}(2)$ (for a detailed discussion of the question, see [13]).

This consideration becomes easier in the limit $M \rightarrow \infty$, i.e., in the case of boundary conditions (10),

$$
R^{+}=q^{-1 / 4}\left(\begin{array}{cccc}
q^{1 / 2} & 0 & 0 & 0 \\
0 & 0 & 1 & 0 \\
0 & 1 & q^{1 / 2}-q^{-1 / 2} & 0 \\
0 & 0 & 0 & q^{1 / 2}
\end{array}\right)
$$

and (38) coincides with the $S L_{q}(2)$ quantum $R$-matrix, which satisfies the braid relation

$$
R_{12} R_{23} R_{12}=R_{23} R_{12} R_{23} .
$$

Now the index $i=1,2\left(V_{1}=a, V_{2}=b\right)$ becomes a genuine quantum group index.

\section{b) Quantum Exchange Algebra for $g(x)(M=\infty)$}

The problem we are going to discuss now can be formulated with the following question: is it possible to define the quantum operators $a, b, c$, and $d$ so that relation (35) will be satisfied for the whole matrix $g(x)$, i.e.

$$
g(x) \otimes g(y)=P g(y) \otimes g(x)\left[R^{+} \theta(x-y)+R^{-} \theta(y-x)\right],
$$

this is a quantum analog of relation (13).

The classical expression for $g(x)$ is (for $M=\infty$ )

$$
g(x)=\left(\begin{array}{cc}
e^{-\phi(x)} & e^{-\phi(x)} \int_{0}^{x} \omega(z) e^{2 \phi(z)} d z \\
\chi(x) e^{-\phi(x)} & e^{-\phi(x)} \chi(x) \int_{0}^{x} \omega(z) e^{2 \phi(z)} d z+e^{\phi(x)}
\end{array}\right)
$$

From the point of view of operator ordering, the problem is only with the operator $d$, where the interchange of ordering of $\chi$ and $\omega$ change the coefficient beyond the second term: $e^{\phi}$. Only this coefficient can be renormalized in the quantum version. Let us mention that the operator $e^{\phi}$ must be understood here as

$$
e^{-\phi(x)} e^{2 \phi(x)}=e^{2 \phi(x)} e^{-\phi(x)}
$$

Then the anomalous dimensions of both first and second terms in $d$ are the same. During the calculation we will use the relation (33) and

$$
\chi(x) \omega(y)-\omega(y) \chi(x)=-\frac{2 \pi i}{K} \delta(x-y) .
$$


Now defining $d$ as:

$$
d=\chi(x) e^{-\phi(x)} \int_{0}^{x} w(z) e^{2 \phi(z)} d z+\alpha e^{-\phi(x)} e^{2 \phi(x)},
$$

we can check the quantum relation (40) is satisfied for the $\alpha$ :

$$
\alpha=\frac{2 \pi i}{K\left(q^{1 / 2}-q^{1 / 2}\right)} .
$$

$g(x)$ with quasiperiodic boundary conditions (18) also satisfies (40) with $R^{+}(M)$ from (35) and corresponding renormalization of the coefficient in the second term in $d: 1 \rightarrow \alpha$.

In the classical limit, $K \rightarrow \infty$ and

$$
\alpha=\frac{2 \pi i}{K\left(e^{\frac{\pi i}{K+2}}-e^{-\frac{\pi i}{K+2}}\right)} \rightarrow 1
$$

At the same time, to the order $O\left(\frac{1}{K}\right), \alpha \rightarrow \frac{K+2}{K} \equiv \alpha_{q, c l}$. Quasiclassical approximation is useful for deriving the relation between the matrix elements of $g(x)$ in (41) and Kac-Moody currents. Therefore in the next section we will assume that $\alpha=\alpha_{q, c l}$.

\section{c) Expressions for Kac-Moody Currents}

The generators of $S L(n)$ Kac-Moody algebra in the free field parametrization as was shown in $[6,8,15-18]$ are polynomials of free fields. Originally for the $S L(2)$ current algebra this parametrization was introduced by Wakimoto [15], therefore we have used the terminology Wakimoto currents:

$$
\begin{gathered}
J^{+}=K \omega, \\
J^{0}=-K \chi \omega+(K+2) \partial \phi, \\
J^{-}=+K \chi^{2} \omega-2(K+2) \chi \partial \phi+K \partial \chi .
\end{gathered}
$$

There are two possible derivations of the relation between currents (45) and matrix elements of quantum field $g(x)$ from $(40)$ :

1. We can check that the Knizhnik-Zamolodchikov [24] relation

$$
(K+2) \partial g=: J g \text { : }
$$

is satisfied;

2. We can define a quantum version of classical formula

$$
J=K \partial g \cdot g^{-1} \text {. }
$$

In the first case it is easy to rewrite the differential $(K+2) \partial g$ in terms of quantum field $g(x)$ itself $(K+2) \partial g=X \cdot g$ and after the normal ordering in the right-hand side (for $\alpha=\alpha_{q, c l}$ ) we obtain that $X$ is exactly Wakimoto's current (45). 
The second case is more complicated. Let us first consider the simple case $J_{+}$. The classical relation is

$$
J_{+}=K\left(a^{\prime} b^{\prime}-b^{\prime} a\right) .
$$

But it can be shown that the right quantum version is

$$
K\left(q^{1 / 2} a^{\prime} b-b^{\prime} a\right)=e^{-\phi} e^{-\phi} e^{2 \phi} K \omega(x)=N K \omega(x),
$$

where we introduced the infinite constant $N$ for the $e^{-\phi(x)} e^{-\phi(x)} e^{2 \phi(x)} \equiv N$. This correction is related to the construction of the quantum inverse operator for $g$ (see below). Moreover, it can be shown in the order $O\left(\frac{1}{K}\right)$ that similar formulas are valid for $J_{-}$and $J_{0}$. Here we must use the relation

$$
\int_{0}^{x} \omega(z) e^{2 \phi(z)} d z \cdot \chi(x)=\chi(x) \int_{0}^{x} \omega(z) e^{2 \phi(z)} d z+\frac{\pi i}{K} e^{2 \phi(x)},
$$

i.e., we assume that $\int_{0}^{x} \delta(z-x) d z=\frac{1}{2}$.

Thus the quantum version of the classical relation $J=K \partial g \cdot g^{-1}$ is

$$
J=K \partial g \cdot\left(g^{-1}\right)_{q} \cdot N
$$

with

$$
\begin{gathered}
\left(g^{-1}\right)_{q}=\left(\begin{array}{cc}
d q^{1 / 2} & -b q^{1 / 2} \\
-c & a
\end{array}\right)=\left(\begin{array}{cc}
q^{1 / 2} & 0 \\
0 & 1
\end{array}\right) \cdot g^{-1} \\
g^{-1}=\left(\begin{array}{cc}
d & -b \\
-c & a
\end{array}\right) .
\end{gathered}
$$

This result is "quasiclassical," but the $\frac{1}{K}$ approximation feels the difference between $K$ and $(K+2)$. More precisely, let us redefine $\left(g^{-1}\right)_{q}$ :

$$
\left(\tilde{g}^{-1}\right)_{q}=N \frac{K}{K+2}\left(g^{-1}\right)_{q}
$$

then

$$
J=(K+2) \partial g \cdot\left(\tilde{g}^{-1}\right)_{q} .
$$

It is now clear that $g \cdot\left(\tilde{g}^{-1}\right)_{q}=1$. The renormalization (50) is related to the fact that the quantum determinant of $g$ is not equal to 1 , but

$$
\operatorname{det}_{q} g=q^{1 / 2} a d-b c=\alpha N \sim \frac{K+2}{K} N,
$$

and therefore it is necessary to include it in the definition of inverse operator. Our derivation of relations (50) and (46) is "quasiclassical," but we think that there must exist an exact quantum derivation; we hope to discuss this question in a separate publication. Let us only mention here that $R(x, y)=R^{+} \theta(x-y)+R^{-} \theta(y-x)$ can be written à la Drinfeld [1] as

$$
R(x ; y)=F^{21} \exp \left\{\frac{\pi i}{K} C \varepsilon(x-y)\right\}\left(F^{12}\right)^{-1}
$$


with constant matrix $F^{12} ; F^{21}=P F^{12} P$. Using this form it must be quite easy to calculate the commutator $\left[g(x)^{\otimes}, \partial g(y)\left(\tilde{g}^{-1}(y)\right)_{q}\right]$. (Details will be published separately [28]).

\section{Quantum Group as a Right-Hand Symmetry in the WZNW Model}

Now, when we have the realization of the quantum group exchange algebra, we can construct the quantum analog of the relations (27), (28) and introduce the quantum group $S L_{q}(2)$ as a right-hand symmetry. (In this section all constructions are for $M=\infty$.) The general picture is as follows: the matrix $g(x)$ contains two columns, corresponding to the spin $\frac{1}{2}$ representation $V_{1}, V_{2}$ of the Kac-Moody group; these two representations are labeled by $\phi$ charge: $V_{1}$ has charge -1 , and $V_{2}$ has +1 ; thus $g(x)$ defines the space $\mathscr{H}=V_{-1}^{1 / 2} \oplus V_{+1}^{1 / 2}$. Defining $g^{j}(x)$ for the $\operatorname{spin} j$ representation of Kac-Moody algebra as a $(2 j+1) \times(2 j+1)$ matrix with matrix elements symmetric polynomials of those for $\operatorname{spin} \frac{1}{2}, g^{j}(x)$ will correspond to the space $\mathscr{H}^{j}=\bigoplus_{\alpha=1}^{2 j+1} V_{\alpha}^{j}$. In this section we demonstrate that the quantum group $S L_{q}(2)$ acts in this space $\mathscr{H}$; it means that the loop group element $g(x)$ defines (for $M \rightarrow \infty$ ) "vertex-chiral-vertex operators" in the terminology of [13].

Let us rewrite (40) in a different basis of a tensor product in the right-hand side:

$$
g_{2}(y) \otimes g_{1}(x)=g(y) \widetilde{\otimes} g(x)=P g(y) \otimes g(x) P,
$$

where $P$ is a permutation matrix. Usually the terminology in the theory of quantum groups as follows: the multipliers in the tensor product are denoted by the label, i.e., $g_{1} \otimes h_{2}$ means that $g$ stands on the left-hand side and $h$ on the righthand side (matrix elements of $g$ and $h$ are $q$-numbers) and $g$ is first in the tensor product; on the other hand, $g_{2} \otimes h_{1}$ means that $g$ is on the left-hand side but $h$ is first in the tensor product. In this terminology we have from (40),

$$
\begin{aligned}
g_{1}(x) \otimes g_{2}(y) & =g_{2}(y) \otimes g_{1}(x)\left[P R^{+} \theta(x-y)+P R^{-} \theta(y-x)\right] \\
& =g_{2}(y) \otimes g_{1}(x)\left[R_{12} \theta(x-y)+R_{21}^{-1} \theta(y-x)\right] .
\end{aligned}
$$

Here $R_{12}=P R^{+}, R_{21}=P R_{12}^{-1} P$. Matrix $R_{12}$ instead of braid relations (39) now satisfies the Yang-Baxter equation [which is equivalent to the associativity of $(51)$ ]

$$
R_{12} R_{13} R_{23}=R_{23} R_{13} R_{12} \text {. }
$$

Now let us consider the quantum analog of the right-hand symmetry $g(x)$ $\rightarrow g(x) \cdot h$, where matrix elements of $g$ and $h$ are commuting: $\left[g_{i j}, h_{k l}\right]=0 ; h \in G$. The condition of invariance of (51) under this transformation is equivalent to

$$
R_{12} h_{1} \otimes h_{2}=h_{2} \otimes h_{1} R_{12} \text {. }
$$

This means that matrix elements of $h \in G=S L(2)$ are $q$-numbers and instead of $S L(2)$ we have $S L_{q}(2)$ (see e.g., [25]); (53) is a quantum analog of Skylanin's brackets. It is no wonder due to general principle [20].

As was demonstrated in the previous section, for the definition of quantum currents we need the inverse operator $\left.\tilde{g}^{-1}\right)_{q}$. At the classical level, the right-hand transformation of $g(x)$ is left-handed for $\left(g^{-1}(x)\right)$

$$
g(x) \rightarrow g(x) \cdot h, \quad g^{-1}(x) \rightarrow h^{-1} \cdot g^{-1}(x) .
$$


For the quantum version of (54) we need to introduce the quantum analog of $h^{-1}$. Such an analog in quantum group literature is known as the antipode $S(h)$ of $h$

$$
h=\left(\begin{array}{ll}
x & y \\
z & t
\end{array}\right) \Rightarrow S(h)=\left(\begin{array}{cc}
t & -q^{-1 / 2} y \\
-q^{1 / 2} z & x
\end{array}\right),
$$

if $\operatorname{det}_{q} h=x t-q^{1 / 2} y z=1$. It is easy to show that $\left(\tilde{g}^{-1}(x)\right)_{q}$ defined by (50) has the property

$$
\left(\tilde{g}^{-1}\right)_{q} \rightarrow S(h)\left(\tilde{g}^{-1}\right)_{q}
$$

when $g(x) \rightarrow g(x) \cdot h$. This means that Wakimoto's currents (50) are invariant under the action of the quantum group [i.e. (50) is an exact quantum formula]

$$
J=(K+2) \partial g \cdot\left(\tilde{g}^{-1}\right)_{q} \rightarrow(K+2) \partial g \cdot h \cdot S(h) \cdot\left(\tilde{g}^{-1}\right)_{q}=J
$$

because of $h \cdot S(h)=S(h) \cdot h=1$.

Thus the quantum group is the right-hand symmetry of the WZW Model.

Acknowledgements. We would like to thank M. Semenov-Tian-Shansky, F. Smirnov, L. Takhtajian, and V. Volkov for stimulating discussions. We are especially grateful to L. Faddeev since many problems addressed in the paper arose in discussions with him. S. Sh. would like to thank J.-L. Gervais, D. Kazhdan, E. Martinec, G. Moore, A. Morozov, P. Nelson, A. Schwartz, and $\mathrm{H}$. Verlinde for interesting discussions. This work was written during the visit of S. Sh. to the United States in Oct.-Dec. 1989, and he thanks G. Moore, D. Gross, and H. Verlinde, and E. Martinec for their great hospitality, correspondingly at Yale University, at Princeton University and at the Enrico Fermi Institute.

Note added. When the present work was finished, J.-L. Gervais informed one of us (S. Sh.) that calculations similar to ones in Sect. 3 were performed for $W$-algebras, and for higher spins in the Liouville theory [26]. Also, L. Faddeev has achieved progress in understanding the quantum group action in the case of $S U(2)$ [27].

\section{References}

1. Tsuchiya, A., Kanie, Y.: In: Conformal field theory and solvable lattice models. Adv. Stud. Pure Math. 16, 297 (1988); Lett. Math. Phys. 13, 303 (1987)

Kohno, T.: Quantized universal enveloping algebras and monodromy of braid groups. In: Braids Contemp. Math., 78. Nagoya preprint, 1988

Fröhlich, J.: Statistics of fields, the Yang-Baxter equation and the theory of knots and links. Lectures at Cargese 1987, to appear in Nonperturbative Quantum Field Theory. New York: Plenum Press

Rehren, K.-H., Schroer, B.: Einstein causality and artin braids. FU preprint 88-0439

Drinfeld, V.G.: Quasi-Hopf algebras and Knizhnik-Zamolodchikov equations. Kiev preprint ITP-89-43E

2. Drinfeld, V.G.: Proc. of Int. Cong. of Math., 1986, p. 798

Jimbo, M.: Lett. Math. Phys. 10, 63 (1985)

Faddeev, L., Reshetikhin, N.Yu., Takhtajian, L.A.: Quantization of Lie groups and Lie algebras. LOMI preprint, E-14-87, Algebra i Analiz 1, 37 (1989) (in Russian)

3. Moore, G., Seiberg, N.: Commun. Math. Phys. 123, 77 (1989)

Moore, G., Seiberg, N.: Lectures on RCFT. Preprint RU-89-32 YCTP-P13-89

4. Witten, E.: Commun. Math. Phys. 121, 351 (1989)

Witten, E.: Gauge theories and integrable lattice models. IAS preprint IASSNS-HEP-89/11

5. Alekseev, A., Shatashvili, S.: Mod. Phys. Lett. A 16, 1551 (1988)

Alekseev, A., Faddeev, L., Shatshvili, S.: Quantization of the symplectic orbis of the compact Lie group by means of the functional integral. Preprint LOMI 1988. J. Geom. Phys. (in press) 
6. Alekseev, A., Shatashvili, S.: Nucl. Phys. B 33, 719 (1989)

7. Alekseev, A., Shatashvili, S.: From geometric quantization to conformal field theory. Commun. Math. Phys. 128, 197-212 (1990)

8. Gerasimov, A., Marshakov, A., Morozov, A., Olshanetsky, M., Shatashvili, S.: Wess-Zumino model as a theory of free fields, I, II, III, IV. Preprints ITEP-64-89, ITEP-70-89, ITEP-72-89, ITEP-74-89

9. Gervais, J.-L., Neveu, A.: Nucl. Phys. B 238, 125 (1984); B 224, 329 (1983)

10. Babelon, O.: Phys. Lett. B 215, 523 (1988)

11. Faddeev, L., Takhtajan, L.: Liouville model on the lattice. Lecture Notes in Phys. Vol. 246, p. 166. Berlin, Heidelberg, New York: Springer 1986

12. Belavin, A.A.: $\mathrm{KdV}$ equation and $W$-algebras. Lecture given at Taniguchi Foundation Symposium. Integrable models in quantum field theory and statistical physics Kyoto, 1988 Bershadsky, M., Ooguri, H.: Hidden $S L(n)$ symmetry in conformal field theories. Commun. Math. Phys. 126, 49-83 (1989)

13. Moore, G., Resketikhin, N.: A comment on quantum group symmetry in conformal field theory. Preprint IASSNS-HEP-89/18

14. Witten, E.: Gauge theories, vertex models, and quantum group. IASSNS-HEP-89/32

15. Wakimoto, M.: Commun. Math. Phys. 104, 605 (1986)

16. Feigin, B., Frenkel, E.: Usp. Mat. Nauk 43, 227 (1988)

17. Zamolodchikov, A.: Unpublished

18. Dotsenko, V.: Unpublished

Bernard, D., Felder, G.: Fock representations and BRST cohomology in $S L(2)$ current algebra, ETH-TH/89-26

Nemeschanky, D.: Feigin-Fuks representation of SU(2) Kac-Moody algebra/USC-89/012 Gawedzki, K.: Quadrature of conformal field theories. IHES preprint 1989

Distler, J., Qiu, Z.: BRS cohomology and a Feigin-Fuks representation ... CLNS-89/911

19. Block, B.: Classical exchange algebras in the Wess-Zumino-Witten model. Tel-Aviv preprint TAUP 1753-89

20. Semenov-Tian-Shansky, M.: Publ. RIMS Kyoto University 21, 1237 (1985)

21. Sklyanin, E.: J. Sov. Math. 19, 1546 (1982)

22. Kirillov, A., Reshetikhin, N.: LOMI preprint E-9-88

23. Felder, G., Fröhlich, J., Keller, G.: Braid matrices and structure constants for minimal models, Zürich preprint, 1989

24. Knizhnik, V., Zamolodchikov, A.: Nucl. Phys. B 247, 83 (1989)

25. Faddeev, L.: Quantum groups, Leningrad preprint, 1989

26. Cremmer, E., Gervais, J.-L.: The quantum group structure associated with non-linearly extended Virasoro algebra, LPTENS-89/19

J.-L. Gervais: The quantum group structure of 2D gravity and minimal models, LPTENS$89 / 14$

27. Faddeev, L.: On the exchange matrix for WZNW model. 132, 131-138 (1990)

28. Alekseev, A.: To be published

Communicated by N. Yu. Reshetikhin 